\title{
Molecular characterisation of the Chlamydia pecorum plasmid from porcine, ovine, bovine, and koala strains indicates plasmid-strain co-evolution
}

Martina Jelocnik, Nathan L Bachmann, Helena Seth-Smith, Nicholas R Thomson, Peter Timms, Adam M Polkinghorne

Background. Highly stable, evolutionarily conserved, small, non-integrative plasmids are commonly found in members of the Chlamydiaceae and, in some species, these plasmids have been strongly linked to virulence. To date, evidence for such a plasmid in Chlamydia pecorum has been ambiguous. In a recent comparative genomic study of porcine, ovine, bovine, and koala C. pecorum isolates, we identified plasmids ( $\mathrm{pCpec}$ ) in a pig and three koala strains, respectively. Screening of further porcine, ovine, bovine, and koala $C$. pecorum isolates for $p C p e c$ showed that $p C p e c$ is common, but not ubiquitous in $C$. pecorum from all of the infected hosts.

Methods. We used a combination of (i) bioinformatic mining of previously sequenced $C$. pecorum genome data sets and (ii) pCpec PCR-amplicon sequencing to characterise a further 17 novel pCpecs in C. pecorum isolates obtained from livestock, including pigs, sheep, and cattle, as well as those from koala.

Results and discussion. This analysis revealed that $\mathrm{pCpec}$ is conserved with all eight coding domain sequences (CDSs) present in isolates from each of the hosts studied. Sequence alignments revealed that the 21 pCpecs show 99\% nucleotide sequence identity, with 83 single nucleotide polymorphisms (SNPs) shown to differentiate all of the plasmids analysed in this study. SNPs were found to be mostly synonymous and were distributed evenly across all eight pCpec CDSs as well as in the intergenic regions. Although conserved, analyses of the $21 \mathrm{pCpec}$ sequences resolved plasmids into 12 distinct genotypes, with five shared between pCpecs from different isolates, and the remaining seven genotypes being unique to a single $\mathrm{pCpec}$. Phylogenetic analysis revealed congruency and co-evolution of pCpecs with their cognate chromosome, further supporting polyphyletic origin of the koala C. pecorum. This study provides further understanding of the complex epidemiology of this pathogen in livestock and koala hosts and paves the way for studies to evaluate the function of this putative $C$. pecorum virulence factor. 
1 Molecular characterisation of the Chlamydia pecorum plasmid from porcine, ovine, bovine,

2 and koala strains indicates plasmid-strain co-evolution

3

4 Martina Jelocnik ${ }^{1}$, Nathan L. Bachmann ${ }^{1}$, Helena Seth-Smith ${ }^{2}$, Nicholas R. Thomson ${ }^{3,4}$, Peter

5 Timms $^{1}$, Adam Polkinghorne ${ }^{1 *}$

6

$7{ }^{1}$ Centre for Animal Health Innovation, University of the Sunshine Coast, Sippy Downs,

8 Queensland 4558, Australia

$9 \quad{ }^{2}$ Functional Genomics Center Zurich, University of Zurich, 8057 Zurich, Switzerland

$10{ }^{3}$ Infection Genomics, The Wellcome Trust Sanger Institute, Hinxton, Cambridge, UK

$11{ }^{4}$ Department of Pathogen Molecular Biology, The London School of Hygiene and Tropical

12 Medicine, London, UK

15 *To whom correspondence should be addressed. Tel: +61 75456 5578; Email:

16 apolking@usc.edu.au 


\section{Abstract}

\section{Background}

19 Highly stable, evolutionarily conserved, small, non-integrative plasmids are commonly found in members of the Chlamydiaceae and, in some species, these plasmids have been strongly linked

21 to virulence. To date, evidence for such a plasmid in Chlamydia pecorum has been ambiguous.

22 In a recent comparative genomic study of porcine, ovine, bovine, and koala C. pecorum isolates,

23 we identified plasmids ( $\mathrm{pCpec}$ ) in a pig and three koala strains, respectively. Screening of further

24 porcine, ovine, bovine, and koala $C$. pecorum isolates for $\mathrm{pCpec}$ showed that $\mathrm{pCpec}$ is common,

25 but not ubiquitious in C. pecorum from all of the infected hosts.

\section{Methods}

27 We used a combination of (i) bioinformatic mining of previously sequenced C. pecorum genome 28 data sets and (ii) pCpec PCR-amplicon sequencing to characterise a further 17 novel pCpecs in C. pecorum isolates obtained from livestock, including pigs, sheep, and cattle, as well as those from koala.

\section{Results and discussion}

This analysis revealed that $\mathrm{pCpec}$ is conserved with all eight coding domain sequences (CDSs) present in isolates from each of the hosts studied. Sequence alignments revealed that the 21 pCpecs show 99\% nucleotide sequence identity, with 83 single nucleotide polymorphisms (SNPs) shown to differentiate all of the plasmids analysed in this study. SNPs were found to be mostly synonymous and were distributed evenly across all eight $\mathrm{pCpec}$ CDSs as well as in the

37 intergenic regions. Although conserved, analyses of the $21 \mathrm{pCpec}$ sequences resolved plasmids 
38 into 12 distinct genotypes, with five shared between $\mathrm{pCpecs}$ from different isolates, and the

39 remaining seven genotypes being unique to a single $\mathrm{pCpec}$. Phylogenetic analysis revealed

40 congruency and co-evolution of $\mathrm{pCpecs}$ with their cognate chromosome, further supporting

41 polyphyletic origin of the koala C. pecorum.

42 This study provides further understanding of the complex epidemiology of this pathogen in

43 livestock and koala hosts and paves the way for studies to evaluate the function of this putative

44 C. pecorum virulence factor. 


\section{Introduction}

Chlamydial plasmids are often referred to as virulence plasmids. They are small, highly conserved, non-integrative and non-conjugative plasmids that are not known to carry genetic cargo such as antibiotic resistance genes. Still, these enigmatic plasmids appear to be an essential part of the genome of the majority of species in the family Chlamydiaceae, with plasmids almost ubiquitously found in human-specific species such as $C$. trachomatis as well as in those that infect animals such as C. psittaci, C. caviae, C. felis, C. muridarum, and C. pneumoniae (Harris et al. 2012; Nunes \& Gomes 2014; Pickett et al. 2005; Read et al. 2013; Rockey 2011; Thomas et al. 1997). The exceptions to the above are (a) the related integrated plasmid in C. suis, which has been shown to be able to carry a tetracycline resistance (tetC) gene (Dugan et al. 2004), (b) $C$. abortus, which is not known to possess any plasmids (Sait et al. 2011) and; (c) very rare human naturally occurring plasmid-free $C$. trachomatis isolates (Peterson et al. 1990; Stothard et al. 1998).

An increasing number of studies have linked the chlamydial plasmid to the pathogenic potential of an infecting isolate, as well as to disease outcome. In animal models, it was demonstrated that naturally occurring plasmid-free $C$. trachomatis and/or plasmid-cured C. muridarum isolates were less infective and less virulent than the wild type plasmid positive ones (Russell et al. 2011; Sigar et al. 2014). Further, C. muridarum studies also demonstrated the critical role of the plasmid in the development and severity of intrauterine infections (Chen et al. 2015; Liu et al. 2014). The utility of the chlamydial plasmid encoded proteins has also been explored as targets for vaccine and diagnostic test development. Plasmid-cured C. trachomatis strains have shown potential as live attenuated vaccines against ocular chlamydial infections in primate models, by providing a complete protection against challenge by a virulent plasmid bearing strain (Kari et al. 
2011). For diagnostic purposes, the plasmid secreted Pgp3 protein has been explored as a marker of chlamydial infections for both humans and animals (Donati et al. 2009; Li et al. 2008), while the $C$. trachomatis specific plasmid sequence was used as a target for commercial molecular diagnostic test for the $C$. trachomatis infections. However, the emergence of new Swedish $C$. trachomatis variants with deletion in the plasmid target sequence evaded diagnostics (Ripa \& Nilsson 2007), highlighting importance of the knowledge of chlamydial plasmid sequences and structure (Seth-Smith et al. 2009).

To date, evidence for a C. pecorum plasmid has been scarce. Recent ovine, bovine, and koala $C$. pecorum whole genome sequencing studies did not report the presence of a plasmid in any of the resulting genome sequences (Bachmann et al. 2014; Bachmann et al. 2015; Mojica et al. 2011; Sait et al. 2014). In our latest comparative genomics study of $C$. pecorum from a variety of hosts, we identified four complete C. pecorum plasmids ( $\mathrm{pCpec}$ ) in the genomes of a porcine and three koala $C$. pecorum isolates, using a set of nine available previously sequenced porcine, ovine, bovine and koala C. pecorum genome data sets (Jelocnik et al. 2015a). Sequence analysis showed that all four $\mathrm{pCpecs}$ were $7.5 \mathrm{kbp}$ in length with eight predicted CDSs with $99 \%$ nucleotide sequence identity, and an overall nucleotide sequence identity of $67-70 \%$ to orthologous genes from chlamydial plasmids in different species (Jelocnik et al. 2015a). A subsequent PCR-based $\mathrm{pCpec}$ screening of 114 C. pecorum strains from pigs, sheep and cattle, and 113 strains from koalas revealed that $\mathrm{pCpec}$, while present in C. pecorum taken from all of the infected hosts, is not ubiquitous in all C. pecorum isolates: $\mathrm{p}$ Cpec was detected in $38.6 \%$ of the sampled C. pecorum taken from livestock, while $\mathrm{pCpec}$ was more commonly detected in the Australian koala C. pecorum isolates, with a 55.8\% detection rate (Jelocnik et al. 2015a). This 
92 varying $\mathrm{pCpec}$ distribution in koala and livestock $C$. pecorum strains potentially sets this plasmid 93 apart from those described in other chlamydial species.

94 In the current study, we fully sequenced and characterised 17 novel pCpecs from a set of $16 C$.

95 pecorum strains from the most common hosts of this pathogen (pigs, sheep, cattle and koalas) in

96 order to examine the genetic structure and diversity of $\mathrm{pCpec}$. In doing so, we observed that,

97 although conserved, the $\mathrm{pCpec}$ sequences are genetically diverse, while the $\mathrm{pCpecs}$ phylogenies

98 indicated congruency and co-evolution with its cognate C. pecorum chromosome.

99

100

Methods and materials

C. pecorum positive clinical samples and isolates used for $\mathrm{p} C p e c$ characterisation and analyses

A total of 17 novel pCpec were characterised from a small collection of cultured C. pecorum isolates and C. pecorum positive clinical swab/tissue samples from two porcine, four ovine, three bovine, and seven koala hosts, previously screened positively for $\mathrm{pCpec}$ presence. $C$. pecorum samples used in the present study and their descriptions are outlined in Table 1.

\section{Chlamydial sequences used for phylogenetic analyses in the present study}

In the present study, we have also used publicly available: (i) plasmid sequences of $C$.

111 (CP000052); C. avium p10DC88 (CPOO6571); C. felis pCfe1 (AP006862); C. psittaci p6BC

112 (CP002550); C. caviae pCpGP 1 (AE015926); and C. pecorum pCpecL1 (KT223773), and (ii) 
113 16S rRNA gene sequences of the corresponding plasmid-bearing chlamydial strains: $C$.

114 pneumoniae LPCoLN (FJ236984); C. muridarum MoPn (CP007217); C. trachomatis A/HAR-13

115 (NR025888); C. avium 10DC88 (NR121781); C. felis Fe/C56 (NC007899); C. psittaci 6BC

116 (NR102492); C. caviae GPIC (NR036833); and C. pecorum L1 (LFRH01000000) for sequence

117 and the Bayesian phylogenetic analyses.

pCpec characterisation from $C$. pecorum whole genome sequencing data

The raw Illumina MiSeq reads of five unpublished koala C. pecorum genomes (HazBoEye, HazBoUGT, NoHerEye, TedHUre, and PMHaUre), sequenced at Wellcome Trust Sanger Institute, Cambridge, UK, were mapped to the newly identified $\mathrm{p} C p e c \mathrm{~L} 1$, and $\mathrm{p} C p e c$ Marsbar sequences(Jelocnik et al. 2015a). Reads mapping to $\mathrm{pCpecL1}$ or $\mathrm{p} C p e c$ Marsbar were assembled from the data sets of five koala $C$. pecorum isolates. The resulting assemblies described a $\sim 7.5$ kbp plasmid, each composed of a single contig (see Table 1). Assembled plasmids were aligned with the porcine $\mathrm{p} C p e c \mathrm{~L} 1$ and koala $\mathrm{pCpecMarsbar}$ using ClustalX, as implemented in Geneious 7.1.4 (Kearse et al. 2012).

\section{Primers design for $\mathrm{pCpec}$ amplicon sequencing}

Using the $\mathrm{p} C p e c \mathrm{~L} 1$ and koala $\mathrm{p} C p e c$ sequences, we designed 23 oligonucleotide PCR primers to

131 amplify overlapping pCpec fragments (Figure 1 and Table S1). Primers were tested for DNA

132 base mismatches using Basic Local Alignment Search Tool (BLAST)

133 (http://blast.ncbi.nlm.nih.gov/Blast.cgi\#), as well as analysed in OligoAnalyzer 3.1 online tool 
134 (https://sg.idtdna.com/calc/analyzer). Primers were designed to have similar annealing

135 temperatures so that they could be used in various combinations to amplify products of between

$136 \sim 700 \mathrm{bp}$ and $3.4 \mathrm{kbp}$ fragments (e.g. PG6 For and PG8 Rev: 3.4 kbp fragments; PG3 For and

137 PGP3 Rev: 731 bp; Table S1).

138 A subset of 12 C. pecorum positive DNA samples (Table 1), obtained from various anatomical

139 sites from porcine, ovine, bovine, and koala hosts were used for further plasmid identification

140 and characterisation. These samples were part of the 227 sample collection previously screened

141 for $\mathrm{pCpec}$ presence (Jelocnik et al. 2015a), by our pCpec-specific PCR. PCR amplifications for

$142 \mathrm{pCpec}$ fragments up to $1 \mathrm{kbp}$ were performed as previously described (Jelocnik et al. 2015a),

143 with appropriate annealing temperature (Table S1). For the amplification of fragments $(>1.5$

$144 \mathrm{kbp}$ ) the LongRange PCR kit, Qiagen, Victoria, Australia, was used as per manufacturer

145 instructions. Isolated $\mathrm{p} C p e c$ Marsbar, $\mathrm{p} C p e c \mathrm{IpTaLe,} \mathrm{p} C p e c \mathrm{DbDeUG} \mathrm{DNA}$ from koala $C$.

146 pecorum strains MC/Mars, DBDeUG, and IpTaLE were used as positive controls. Genomic

147 DNA from the non-plasmid containing strains L17 and L71, and dH20 were used as negative

148 controls (Jelocnik et al. 2015a). The presence of the amplicons were confirmed on 1.5\% agarose-

149 TBE gels, and then purified and dideoxy sequenced (The Institute for Future Environments

150 (IFE), Queensland University of Technology (QUT), Brisbane, Australia using the Applied

151 Biosystems ABI3500 Gene analyser).

152 Forward and reverse chromatogram of each sequenced amplicon was aligned in Geneious 7.1.4

153 and the amplicon consensus sequence was extracted. Overlapping amplicon sequences were used

154 to assemble the full length plasmid sequence for each sample. The derived $\mathrm{pCpec}$ sequences

155 were annotated with RAST (Aziz et al. 2008), and further curated in Geneious 7.1.4. pCpecs

156 translated CDSs were further analysed in blastp for comparison 
157 (http://blast.ncbi.nlm.nih.gov/Blast.cgi), as well as Universal Protein Resource (UniProt from

158 http://www.uniprot.org/) and Conserved Domains Database (CDD from

159 http://www.ncbi.nlm.nih.gov/cdd) to assess the protein functionality. Plasmid sequences were 160 deposited in Genbank under accession numbers KT223766 - KT223781, and KT352920 161 KT352924.

\section{1 pCpec sequence and phylogenetic analyses}

In order to assess the evolutionary relationships of $\mathrm{pCpecs}$ and the level of sequence diversity, we determined the number of synonymous $\left(d_{s}\right)$ and non-synonymous $\left(d_{n}\right)$ substitutions found in all 21 C. pecorum plasmid sequences included in this study. The number of polymorphic (segregating) sites, CDS alleles, plasmid genotypes, and putative recombination events were determined using DnaSP 5.0 (Librado \& Rozas 2009). A pCpec ancestral sequence was reconstructed using an alignment of all $21 \mathrm{pCpecs}$ performed on the FastML server (Ashkenazy et al. 2012). Best-fit models of nucleotide substitution used for phylogenetic analysis of the plasmid sequences were estimated by jModelTest v.2.1.1 (Darriba et al. 2012). A rooted Bayesian phylogenetic tree consisting of eight chlamydial plasmids or their corresponding 16SrRNA gene sequences were constructed with MrBayes as implemented in Geneious 7.1.4, with $\mathrm{HKY}+\mathrm{I}+\mathrm{G}$ for plasmid, and $\mathrm{HKY}+\mathrm{G}$ for $16 \mathrm{~S}$ rRNA sequences. Both trees used the $C$. muridarum plasmid or 16S rRNA sequences as a root. A Bayesian phylogenetic tree consisting of all 21 C. pecorum $7.5 \mathrm{kbp}$ plasmid sequences was also constructed with MrBayes using GTR + G model. C. pneumoniae plasmid pLPCoLN sequence was used as an outgroup. The plasmid phylogenetic tree was compared to the chromosome Multi Locus Sequence Typing 
179 (MLST-based) phylogeny constructed from the concatenated sequence of the seven MLST

180 house-keeping (HK) gene fragments (Jelocnik et al. 2013) for corresponding samples, where

181 available (excluding SA/K84/Ure, SA/K09/Ure and Vic/R6/UGT ). A Bayesian MLST

182 phylogenetic tree of 18 C. pecorum samples sequences was also constructed in MrBayes with the

183 HKY 85+I+G model. Run parameters for all phylogenetic trees from this study included four

184 Markov Chain Monte Carlo (MCMC) chains with a 1000000 generations, sampled every 100

185 generations and with the first 10000 trees were discarded as burn-in.

187 Results and Discussion

pCpec: a newly characterised member of chlamydial plasmids

With the recent observation that the $C$. pecorum plasmid is common but not ubiquitous across this species (Jelocnik et al. 2015a), we characterised an additional 17 novel pCpec sequences from C. pecorum strains isolated from a variety of hosts. In total, we included a set of these $21 C$. pecorum plasmid sequences for analyses.

These data revealed that all $21 \mathrm{pCpecs}$ were $7.5 \mathrm{kbp}$ in size with a low $\mathrm{G}+\mathrm{C}$ (av. 31.6\%) content, typical for chlamydial plasmids (Thomas et al. 1997). Using an alignment of eight representative plasmid sequences from related chlamydial species (including $\mathrm{pCpec}$ ), we constructed a plasmid phylogeny and compared it to the $16 \mathrm{~S}$ rRNA gene phylogeny of the corresponding strains carrying these plasmids (Figure S1). As observed in Figure S1 and consistent with previous phylogenetic analysis of chlamydial plasmids from other species (Mitchell et al. 2010), plasmid phylogenies displayed a similar topology to that observed for the $16 \mathrm{~S}$ rRNA derived chlamydial phylogeny. In this study, both C. pecorum pCpecL1 and the C. pecorum 16SrRNA-gene 
201 sequence clustered with its closest chlamydial relative C. pneumoniae (Gupta et al. 2015). The

202 observed phylogenetic relationships of the chlamydial plasmids also suggest that the plasmids

203 evolve in parallel with their bacterial host (Rockey 2011), and therefore that these plasmids were

204 acquired early in the evolution of Chlamydiae and have been subject to little between-species

205 recombination (Andersson \& Kurland 1998), although intra-species plasmid-associated

206 recombination has been reported previously in C. trachomatis (Harris et al. 2012).

pCpec phylogeny

209

Overall, the alignment of the $21 \mathrm{pCpec}$ sequences resolved 12 distinct plasmid sequence types (genotypes), with five genotypes shared between $\mathrm{pCpecs}$ from different isolates, and the

211 remaining seven genotypes were unique to a single $\mathrm{p} C p e c$ (Figure $\mathrm{S} 2$ ). The three porcine $\mathrm{pCpecs}$ were of an identical sequence type (Genotype L). Among the 11 koala pCpecs, we identified five distinct genotypes, with: (i) pCpecs SAK09Ure, SAK84Ure and VicR6UGT sharing the first

214 (Genotype A); (ii) pCpecs NoHerEyes, PMHaUre, TedHUre and DbDeUG sharing the second

215 (Genotype B) and; (iii) pCpecs HazBoEye and HazBoUgt sharing the third genotype (Genotype

216 C); in contrast, the pCpecs Marsbar, and IpTale were of a distinct fourth and fifth genotype each

217 (Genotypes D and E, respectively). With the exception of the pCpecs CurE11Rec, and

218 CurE19Rec which were also of an identical sequence type (Genotype F), the remaining two

219 ovine (W73 and IPA), and the three bovine (WAB31Ileal, 66P130, and LW623) pCpecs were of

220 a unique genotype each (Genotypes G, H, I, J and K, respectively) (Figure S2). 
222 We also compared the phylogenies for our $\mathrm{pCpec}$ sequences (Figure 2A) to the corresponding

223 MLST phylogenetic tree for the strains harbouring these plasmids (Figure 2B). In the absence of

224 whole genome sequences for a number of isolates, MLST-derived phylogenies were utilised, as

225 they have previously been shown to be congruent with those constructed from core genome

226 alignments (Bachmann et al. 2014; Bachmann et al. 2015). Using the C. pneumoniae plasmid

227 sequence as an out-group, the root of the C. pecorum plasmid tree falls between two distinct

228 pCpec clades (Figure S3). Further, the phylogenetic tree also resolved $21 \mathrm{pCpec}$ sequences into

229 lineages according to their genotype and/or closely related genotypes (Figure 2A, S2B). Clade 1

230 included a distinct lineage consisting of $\mathrm{pCpecs}$ from South Australian (SA) and Victorian (Vic)

231 koala strains of an identical genotype, as well as the more diverse lineage that consisted of the

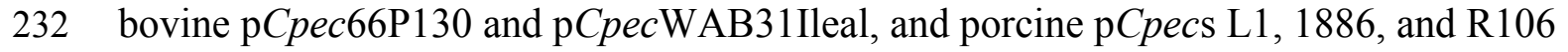

233 sequences (Figure 2A). The genetically diverse Clade 2 included all the remaining eight pCpecs

234 from Queensland (QLD) and New South Wales (NSW) koala C. pecorum strains forming a well-

235 supported lineage, as well as the pCpecs CurE11Rec, CurE19Rec from Australian sheep strains,

236 and pCpecLW623, pCpecW73 and pCpecIPA from USA bovine and ovine C. pecorum isolates,

237 respectively (Figure 2A). pCpec phylogeny was found to be largely congruent with a C. pecorum

238 MLST phylogeny (Figure 2B). In the MLST tree, we also observed two distinct clades. The first

239 clade included lineages from porcine and bovine C. pecorum (Figure 2B), while the second

240 genetically diverse clade included all the koala C. pecorum strains resolved into their own

241 lineages, and the four ovine (W73, CurE11Rec, CurE19Rec, and IPA) and the bovine LW623

242 MLST sequences (Figure 2B). 
244 Overall, this phylogenetic analysis revealed similar levels of inter-host C. pecorum strain genetic

245 variability to that described previously using various other molecular markers (Jelocnik et al.

246 2015b; Mohamad et al. 2014), as well as congruency with our previously published C. pecorum

247 core genome phylogenies (Bachmann et al. 2014; Jelocnik et al. 2015a). The co-evolution of

248 plasmids with the chromosome of $C$. pecorum is consistent with that previously described for $C$.

249 trachomatis (Seth-Smith et al. 2009). Separation of the pCpec sequences into two distinct clades

250 with different lineages suggests that, like the pathogen itself, the current $C$. pecorum plasmids

251 are of a polyphyletic origin. Using pCpecs from koala strains as an example, we observed an

252 evolutionary split between the genetically identical plasmids from South Australian (SA) and

253 Victorian (Vic) koala C. pecorum strains, and the more genetically diverse plasmids from

254 Queensland (QLD) and New South Wales (NSW) koala strains. It should be also noted that the

255 plasmids from QLD and NSW koala strains appear to be more closely related to the plasmids

256 from sheep strains than to plasmids from strains from the same host, an observation that is

257 consistent with our previous molecular typing and comparative genomics studies of these $C$.

258 pecorum strains (Bachmann et al. 2015).

Sequence analyses of pCpecs and its encoded proteins

261 Manually curated annotation of the $21 \mathrm{pCpec}$ sequences revealed a similar structure to that of

262 chlamydial plasmids from other species with eight CDSs predicted in total. CDSs 1, 2, 3, 7 and 8

263 are putatively involved in plasmid maintenance and replication, while CDSs 4, 5 and 6 are

264 associated with chlamydial-specific virulence (Gong et al. 2013; Song et al. 2013; Thomas et al. 
265 1997) (Table 2). pCpec also carry the four $22 \mathrm{bp}$ tandem repeats located in the putative origin of

266 replication (Figure 1, S2A).

267 The alignment of the 21 full length $\mathrm{p} C p e c$ sequences derived either from genome sequencing or

268 PCR-based approaches, revealed from 0 to 83 SNPs (a maximum split between the two pCpecs

269 sequences) distributed evenly around the plasmid. A single insertion of $1 \mathrm{bp}$ was seen in bovine

270 66P130 and LW623, and porcine L1, 1886 and R106 pCpecs located in the intergenic region

271 between CDS 6 and CDS 7 (Figure S2A). Using the reconstructed plasmid sequence as a

272 reference, we also identified pCpec homoplasic SNPs. As observed in the pCpec SNPs-only

273 alignment (Figure S2B), we detected 10 homoplasic SNPs, three of them (at positions 9, 10 and

274 84) in the intergenic regions, while the remaining seven (at positions 1, 16, 29, 69, 75, 76 and 78)

275 were in the $\mathrm{pCpec}$ CDSs 1, 2, 3, 7 and 8. Homoplasic SNPs at positions 1, 16, 76 and 78 also

276 resulted in a non-synonymous amino acid change (Figure S2B). Although the most parsimonious

277 explanation is for putative recombination between these strains, in our present analyses using a

278 set of $21 \mathrm{pCpecs}$ we do not have an evidence of recombination, hence it is uncertain whether the

279 observed homoplasic SNPs occurred as a result of a homologous recombination or by

280 independent selection (Harris et al. 2012).

281 We further assessed each of the eight CDSs as well as the intergenic regions for synonymous and

282 non-synonymous SNPs (Table 2). Most SNPs were detected in the CDS 2 (putative integrase)

283 and CDS 4 (putative plasmid virulence protein), with 13 and 12 SNPs each, respectively. pCpecs

284 CDS 2 also had the most non-synonymous changes $(n=6)$ (Figure S2A) and could be detected in 285 eight different alleles. In contrast to the seven polymorphic CDSs with seven to 13 SNPs each, 286 pCpecs CDS 6 was the most conserved sequence, with only three synonymous SNPs. 
288 The level and distribution of the $\mathrm{pCpec}$ sequence variation was comparable to that in $C$.

289 trachomatis plasmids (Ferreira et al. 2013; Seth-Smith et al. 2009). Using only a limited set of

290 pCpec sequences, in our analyses the sequence variation mostly appears to be a result of

291 synonymous changes. Nevertheless, it was interesting to note that most of the non-synonymous

292 changes, including the ones in virulence-associated CDSs 4 and 5, seem to be accumulating in

293 pCpecs from C. pecorum strains derived from sheep, and QLD and NSW koalas presenting with

294 disease (Figure S2A). In regards to the plasmid virulence-associated CDSs, CDS 4 was the most

295 polymorphic locus with only two non-synonymous changes, however. C. pecorum CDS 5 (pgp3)

296 had the most non-synonymous changes $(n=4)$, mainly accumulating in the 5 'end of the gene,

297 while the CDS 6 was the most conserved. C. trachomatis CDS 5, encoding PgP3, is the most

298 polymorphic CDS in this plasmid (Ferreira et al. 2013; Seth-Smith et al. 2009). Cytosol-exported

299 PgP3 has been implicated as one of the major chlamydial factors affecting disease pathogenesis

300 in C. trachomatis (Li et al. 2008), therefore possibly due to the host's immune pressure, this CDS

301 is under pressure to accumulate SNPs. At present, it is unknown what selective pressures are

302 being placed on the Chlamydia-specific virulence-associated pCpec CDS 4 and 5 genes. Future

303 studies to investigate this will require a larger set of $\mathrm{pCpecs}$ from strains isolated from a variety

304 of diseased as well as healthy hosts, using both bioinformatics as well as cell biology

305 approaches.

306

Detection of the most variation and non-synonymous changes in pCpec CDS 2 contrasts with its strong conservation in the $C$. trachomatis plasmid. The remaining $\mathrm{p} C p e c \mathrm{CDSs}$ displayed high sequence conservation, particularly for CDS 7 and CDS 8. These CDSs are putatively denoted as the partitioning co-transcribing genes (Ferreira et al. 2013), and their encoded proteins may 
310 play essential role in effective plasmid segregation to the progeny cells, hence their sequence

311 conservation is not surprising. Based on our findings, $\mathrm{pCpec}$ sequences appear to be evolutionary

312 conserved and it is likely that the function of the encoded proteins is similar to that previously

313 predicted for C. trachomatis (Seth-Smith et al. 2009), and C. pneumoniae (Thomas et al. 1997).

\section{Conclusions}

316 In the present study, we have characterised the genetic structure and phylogenetic relationships

317 of $21 \mathrm{pCpec}$ from porcine, ovine, bovine, and koala strains. Our data suggests that the pCpec

318 sequence is evolutionarily conserved, as it is in related chlamydial species, with the chlamydial

319 plasmid virulence-associated features present. The pCpec phylogenies revealed "co-evolution"

320 of plasmids with their respective $C$. pecorum chromosomes, further supporting a polyphyletic

321 evolution of this pathogen at least in Australian koalas (Figure 2). Based on the pCpec sequence

322 and predicted gene functions, the level and nature of the plasmid conservation suggests that

$323 \mathrm{pCpec}$, where found, is potentially important for during growth, infection, and/or transmission of

324 the bacterium within a population, as has been suggested in studies comparing plasmid-positive

325 and plasmid-negative chlamydial isolates (Rockey 2011; Russell et al. 2011). Although how this

326 relates to the plasmid-negative $C$. pecorum is not clear at present. This study provides more clues

327 to understand the complex epidemiology of this pathogen in livestock and koala hosts.

329 Competing interests

330 The authors declare that they have no competing interests. 


\section{Acknowledgments}

333 We thank Alyce Taylor-Brown for technical assistance and Tamieka Fraser and Courtney Waugh

334 for sample provision. 
335

336

337

338

339

340

341

342

343

344

345

346

347

348

349

350

351

352

353

354

355

356

357

358

359

360

361

362

363

364

365

366

\section{References}

Andersson SGE, and Kurland CG. 1998. Reductive evolution of resident genomes. Trends in Microbiology 6:263-268. http://dx.doi.org/10.1016/S0966-842X(98)01312-2

Ashkenazy H, Penn O, Doron-Faigenboim A, Cohen O, Cannarozzi G, Zomer O, and Pupko T. 2012. FastML: a web server for probabilistic reconstruction of ancestral sequences. Nucleic Acids Research 40:W580-584. 10.1093/nar/gks498

Aziz RK, Bartels D, Best AA, DeJongh M, Disz T, Edwards RA, Formsma K, Gerdes S, Glass EM, Kubal M, Meyer F, Olsen GJ, Olson R, Osterman AL, Overbeek RA, McNeil LK, Paarmann D, Paczian T, Parrello B, Pusch GD, Reich C, Stevens R, Vassieva O, Vonstein V, Wilke A, and Zagnitko O. 2008. The RAST Server: rapid annotations using subsystems technology. BMC Genomics 9:75. 10.1186/1471-2164-9-75

Bachmann N, Fraser T, Bertelli C, Jelocnik M, Gillett A, Funnell O, Flanagan C, Myers GS, Timms P, and Polkinghorne A. 2014. Comparative genomics of koala, cattle and sheep strains of Chlamydia pecorum. BMC Genomics 15:667.

Bachmann NL, Sullivan MJ, Jelocnik M, Myers GSA, Timms P, and Polkinghorne A. 2015.

Culture-independent genome sequencing of clinical samples reveals an unexpected heterogeneity of infections by Chlamydia pecorum. Journal of Clinical Microbiology 53:1573-1581. 10.1128/jcm.03534-14

Chen J, Yang Z, Sun X, Tang L, Ding Y, Xue M, Zhou Z, Baseman J, and Zhong G. 2015. Intrauterine Infection with plasmid-free Chlamydia muridarum reveals a critical role of the plasmid in chlamydial ascension and establishes a model for evaluating plasmid-independent pathogenicity. Infection and Immunity 83:2583-2592. 10.1128/iai.00353-15

Darriba D, Taboada GL, Doallo R, and Posada D. 2012. jModelTest 2: more models, new heuristics and parallel computing. Nature Methods 9:772-772.

http://www.nature.com/nmeth/journal/v9/n8/abs/nmeth.2109.html\#supplementary-information Donati M, Laroucau K, Storni E, Mazzeo C, Magnino S, Di Francesco A, Baldelli R, Ceglie L, Renzi M, and Cevenini R. 2009. Serological response to pgp3 protein in animal and human chlamydial infections. Veterinary Microbiology 135:181-185. 10.1016/j.vetmic.2008.09.037 Dugan J, Rockey DD, Jones L, and Andersen AA. 2004. Tetracycline resistance in Chlamydia suis mediated by genomic islands inserted into the chlamydial inv-like gene. Antimicrobial Agents and Chemotherapy 48:3989-3995. 10.1128/AAC.48.10.3989-3995.2004 
367 Ferreira R, Borges V, Nunes A, Borrego MJ, and Gomes JP. 2013. Assessment of the load and

368 transcriptional dynamics of Chlamydia trachomatis plasmid according to strains' tissue tropism.

369 Microbiological Research 168:333-339. 10.1016/j.micres.2013.02.001

370 Gong S, Yang Z, Lei L, Shen L, and Zhong G. 2013. Characterization of Chlamydia trachomatis

371 plasmid-encoded open reading frames. Journal of Bacteriology 195:3819-3826.

$37210.1128 / \mathrm{jb} .00511-13$

373 Gupta R, Naushad S, Chokshi C, Griffiths E, and Adeolu M. 2015. A phylogenomic and

374 molecular markers based analysis of the phylum Chlamydiae: proposal to divide the class

375 Chlamydiia into two orders, Chlamydiales and Parachlamydiales ord. nov., and emended

376 description of the class Chlamydiia. Antonie van Leeuwenhoek 108:765-781. 10.1007/s10482-

377 015-0532-1

378 Harris SR, Clarke IN, Seth-Smith HMB, Solomon AW, Cutcliffe LT, Marsh P, Skilton RJ,

379 Holland MJ, Mabey D, Peeling RW, Lewis DA, Spratt BG, Unemo M, Persson K, Bjartling C,

380 Brunham R, de Vries HJC, Morre SA, Speksnijder A, Bebear CM, Clerc M, de Barbeyrac B,

381 Parkhill J, and Thomson NR. 2012. Whole-genome analysis of diverse Chlamydia trachomatis

382 strains identifies phylogenetic relationships masked by current clinical typing. Nature Genetics

383 44:413-419. 10.1038/ng.2214

384 Jelocnik M, Bachmann NL, Kaltenboeck B, Waugh C, Woolford L, Speight K. Natasha, Gillett

385 A, Higgins DP, Flanagan C, Myers Garry S. A., Timms P, and Polkinghorne A. 2015a. Genetic

386 diversity in the plasticity zone and the presence of the chlamydial plasmid differentiates

387 Chlamydia pecorum strains from pigs, sheep, cattle, and koalas. BMC Genomics. 16:893.

388 10.1186/s12864-015-2053-8

389 Jelocnik M, Forshaw D, Cotter J, Roberts D, Timms P, and Polkinghorne A. 2014a. Molecular

390 and pathological insights into Chlamydia pecorum-associated sporadic bovine encephalomyelitis

391 (SBE) in Western Australia. BMC Veterinary Research 10:121. 10.1186/1746-6148-10-121

392 Jelocnik M, Frentiu FD, Timms P, and Polkinghorne A. 2013. Multi-locus sequence analysis

393 provides insights into the molecular epidemiology of Chlamydia pecorum infections in

394 Australian sheep, cattle and koalas. Journal of Clinical Microbiology 51:2625-2632.

$395 \quad 10.1128 / \mathrm{jcm} .00992-13$ 
396 Jelocnik M, Self R, Timms P, Borel N, and Polkinghorne A. 2015b. Novel sequence types of 397 Chlamydia pecorum infect free-ranging Alpine ibex (Capra ibex) and red deer (Cervus elaphus) 398 in Switzerland. Journal of Wildlife Diseases 51:479-483. 10.7589/2014-08-220

399 Jelocnik M, Walker E, Pannekoek Y, Ellem J, Timms P, and Polkinghorne A. 2014b. Evaluation 400 of the relationship between Chlamydia pecorum sequence types and disease using a species401 specific multi-locus sequence typing scheme (MLST). Veterinary Microbiology 174:214-222. 402 10.1016/j.vetmic.2014.08.018

403 Kaltenboeck B, Kousoulas KG, and Storz J. 1993. Structures of and allelic diversity and 404 relationships among the major outer membrane protein (ompA) genes of the four chlamydial 405 species. Journal of Bacteriology 175:487-502.

406 Kari L, Whitmire WM, Olivares-Zavaleta N, Goheen MM, Taylor LD, Carlson JH, Sturdevant 407 GL, Lu C, Bakios LE, Randall LB, Parnell MJ, Zhong G, and Caldwell HD. 2011. A live408 409 attenuated chlamydial vaccine protects against trachoma in nonhuman primates. The Journal of Experimental Medicine 208:2217-2223. 10.1084/jem.20111266

Kearse M, Moir R, Wilson A, Stones-Havas S, Cheung M, Sturrock S, Buxton S, Cooper A, Markowitz S, Duran C, Thierer T, Ashton B, Meintjes P, and Drummond A. 2012. Geneious Basic: an integrated and extendable desktop software platform for the organization and analysis of sequence data. Bioinformatics 28:1647-1649. 10.1093/bioinformatics/bts199

414 Koelbl O. 1969. Untersuchungen ueber das vorkommen von Miyagawanellen beim schwein. 415 Wien Tierarztl Mschr 56:332-335.

416 Li Z, Chen D, Zhong Y, Wang S, and Zhong G. 2008. The chlamydial plasmid-encoded protein 417 pgp3 is secreted into the cytosol of Chlamydia-infected cells. Infection and Immunity 76:3415418 3428. 10.1128/iai.01377-07

419 Librado P, and Rozas J. 2009. DnaSP v5: a software for comprehensive analysis of DNA 420 polymorphism data. Bioinformatics 25:1451-1452. 10.1093/bioinformatics/btp187

421 Liu Y, Huang Y, Yang Z, Sun Y, Gong S, Hou S, Chen C, Li Z, Liu Q, Wu Y, Baseman J, and 422 Zhong G. 2014. Plasmid-encoded Pgp3 is a major virulence factor for Chlamydia muridarum to 423 induce hydrosalpinx in mice. Infection and Immunity 82:5327-5335. 10.1128/iai.02576-14 424 Mitchell C, Hovis K, Bavoil P, Myers G, Carrasco J, and Timms P. 2010. Comparison of koala 425 LPCoLN and human strains of Chlamydia pneumoniae highlights extended genetic diversity in 426 the species. BMC Genomics 11:442. 
427 Mohamad KY, Kaltenboeck B, Rahman Kh S, Magnino S, Sachse K, and Rodolakis A. 2014.

428 Host adaptation of Chlamydia pecorum towards low virulence evident in co-evolution of the

429

430

431

432

433

434

435

436

437

438

439

440

441

442

443

444

445

446

447

448

449

450

451

452

453

454

455

456

457 ompA, incA, and ORF663 Loci. PLoS ONE 9:e103615. 10.1371/journal.pone.0103615

Mojica S, Huot Creasy H, Daugherty S, Read TD, Kim T, Kaltenboeck B, Bavoil P, and Myers GSA. 2011. Genome sequence of the obligate intracellular animal pathogen Chlamydia pecorum E58. Journal of Bacteriology 193:3690. 10.1128/jb.00454-11

Nunes A, and Gomes JP. 2014. Evolution, phylogeny, and molecular epidemiology of

Chlamydia. Infection, Genetics and Evolution 23:49-64.

http://dx.doi.org/10.1016/j.meegid.2014.01.029

Peterson EM, Markoff BA, Schachter J, and de la Maza LM. 1990. The 7.5-kb plasmid present in Chlamydia trachomatis is not essential for the growth of this microorganism. Plasmid 23:144148.

Pickett MA, Everson JS, Pead PJ, and Clarke IN. 2005. The plasmids of Chlamydia trachomatis and Chlamydophila pneumoniae (N16): accurate determination of copy number and the paradoxical effect of plasmid-curing agents. Microbiology 151:893-903. 10.1099/mic.0.27625-0 Read TD, Joseph SJ, Didelot X, Liang B, Patel L, and Dean D. 2013. Comparative analysis of Chlamydia psittaci genomes reveals the recent emergence of a pathogenic lineage with a broad host range. MBio 4. 10.1128/mBio.00604-12

Ripa T, and Nilsson PA. 2007. A Chlamydia trachomatis strain with a 377-bp deletion in the cryptic plasmid causing false-negative nucleic acid amplification tests. Sexually Transmitted Diseases 34:255-256. 10.1097/OLQ.0b013e31805ce2b9

Rockey DD. 2011. Unraveling the basic biology and clinical significance of the chlamydial plasmid. Journal of Experimental Medicine 208:2159-2162. 10.1084/jem.20112088

Russell M, Darville T, Chandra-Kuntal K, Smith B, Andrews CW, Jr., and O'Connell CM. 2011. Infectivity acts as in vivo selection for maintenance of the chlamydial cryptic plasmid. Infection and Immunity 79:98-107. 10.1128/iai.01105-10

Sait M, Clark EM, Wheelhouse N, Livingstone M, Spalding L, Siarkou VI, Vretou E, Smith DGE, Lainson FA, and Longbottom D. 2011. Genome sequence of the Chlamydophila abortus variant strain LLG. Journal of Bacteriology 193:4276-4277. 10.1128/JB.05290-11

Sait M, Livingstone M, Clark EM, Wheelhouse N, Spalding L, Markey B, Magnino S, Lainson FA, Myers GS, and Longbottom D. 2014. Genome sequencing and comparative analysis of three 
458 Chlamydia pecorum strains associated with different pathogenic outcomes. BMC Genomics

459 15:23. 10.1186/1471-2164-15-23

460 Seth-Smith HM, Harris SR, Persson K, Marsh P, Barron A, Bignell A, Bjartling C, Clark L, 461 Cutcliffe LT, Lambden PR, Lennard N, Lockey SJ, Quail MA, Salim O, Skilton RJ, Wang Y, 462 Holland MJ, Parkhill J, Thomson NR, and Clarke IN. 2009. Co-evolution of genomes and 463 plasmids within Chlamydia trachomatis and the emergence in Sweden of a new variant strain. 464 BMC Genomics 10:239. 10.1186/1471-2164-10-239

465 Sigar IM, Schripsema JH, Wang Y, Clarke IN, Cutcliffe LT, Seth-Smith HM, Thomson NR, 466 Bjartling C, Unemo M, Persson K, and Ramsey KH. 2014. Plasmid deficiency in urogenital 467 isolates of Chlamydia trachomatis reduces infectivity and virulence in a mouse model. Pathog 468 Dis 70:61-69. 10.1111/2049-632x.12086

469 Song L, Carlson JH, Whitmire WM, Kari L, Virtaneva K, Sturdevant DE, Watkins H, Zhou B, 470 Sturdevant GL, Porcella SF, McClarty G, and Caldwell HD. 2013. Chlamydia trachomatis 471 plasmid-encoded Pgp4 is a transcriptional regulator of virulence-associated genes. Infection and 472 Immunity 81:636-644. 10.1128/iai.01305-12

473 Stothard DR, Williams JA, Van Der Pol B, and Jones RB. 1998. Identification of a Chlamydia 474 trachomatis serovar E urogenital isolate which lacks the cryptic plasmid. Infection and Immunity 475 66:6010-6013.

476 Thomas NS, Lusher M, Storey CC, and Clarke IN. 1997. Plasmid diversity in Chlamydia.

477 Microbiology 143 (6):1847-1854. 
Table 1. Descriptions of $C$. pecorum samples used for plasmid characterisation.

\begin{tabular}{|c|c|c|c|c|c|c|c|c|}
\hline $\begin{array}{l}\text { Plasmid } \\
\text { (pCpec) ID }\end{array}$ & $\begin{array}{c}\text { Host and } \\
\text { country of } \\
\text { origin }\end{array}$ & $\begin{array}{l}\text { Type of sample/ } \\
\text { Anatomical site }\end{array}$ & $\begin{array}{c}\text { Host } \\
\text { pathology }\end{array}$ & $\begin{array}{l}\text { Plasmid } \\
\text { generation }\end{array}$ & $\begin{array}{l}\text { Length } \\
\text { (bp) }\end{array}$ & $\begin{array}{c}\text { GC content } \\
(\%)\end{array}$ & $\begin{array}{c}\text { Accession } \\
\text { number }\end{array}$ & Strain reference \\
\hline L1* & Pig, Austria & Culture/Lung & Pneumonia & $\mathrm{WGS}^{3}$ & 7548 & 31.7 & KT223773 & Koelbl, 1969 \\
\hline R106 & Pig, Austria & Culture/Lung & Pneumonia & Amplicon seq. ${ }^{4}$ & 7548 & 31.7 & KT223776 & Koelbl, 1969 \\
\hline 1886 & Pig, Austria & Culture/Lung & Pneumonia & Amplicon seq. ${ }^{4}$ & 7548 & 31.7 & KT223767 & Koelbl, 1969 \\
\hline IPA & Sheep, USA & Culture/Joint & Polyarthritis & Amplicon seq. ${ }^{4}$ & 7547 & 31.6 & KT223771 & Bachmann, et al., 2014 \\
\hline W73 & Sheep, Ireland & Culture/Faeces & Asymptomatic & Amplicon seq. ${ }^{4}$ & 7547 & 31.6 & KT223780 & Sait, et al., 2014 \\
\hline Cur/E19/Rec & Sheep, Australia & Swab sample/Rectum & $\mathrm{NCD}^{1}$ & Amplicon seq. ${ }^{4}$ & 7547 & 31.6 & KT223769 & Jelocnik, et al., 2014b \\
\hline Cur/E11/Rec & Sheep, Australia & Swab sample/Rectum & $\mathrm{NCD}^{1}$ & Amplicon seq. ${ }^{4}$ & 7547 & 31.6 & KT223768 & Jelocnik, et al., 2014b \\
\hline LW623 & Cattle, USA & Culture/Brain & Encephalomyelitis & Amplicon seq. ${ }^{4}$ & 7547 & 31.6 & KT223774 & Kaltenboeck, et al., 1993 \\
\hline WA/B31/Ileal & Cattle, Australia & Tissue sample/Ileum & $\mathrm{SBE}^{2}$ & Amplicon seq. ${ }^{4}$ & 7548 & 31.7 & KT223781 & Jelocnik, et al., 2014a \\
\hline $66 \mathrm{P} 130$ & Cattle, USA & Culture/Faeces & $\mathrm{NCD}^{1}$ & Amplicon seq. ${ }^{4}$ & 7548 & 31.7 & KT223766 & Kaltenboeck, et al., 1993 \\
\hline $\mathrm{SA} / \mathrm{K} 84 / \mathrm{Ure}$ & Koala, Australia & Swab sample/Urethra & $\mathrm{NCD}^{1}$ & Amplicon seq. ${ }^{4}$ & 7547 & 31.6 & KT223778 & Jelocnik et al., 2015a \\
\hline $\mathrm{SA} / \mathrm{K} 09 / \mathrm{Ure}$ & Koala, Australia & Swab sample/Urethra & $\mathrm{NCD}^{1}$ & Amplicon seq. ${ }^{4}$ & 7547 & 31.6 & KT223777 & Jelocnik et al., 2015a \\
\hline Vic/R6/UGT & Koala, Australia & Swab sample/UGT & $\mathrm{NCD}^{1}$ & Amplicon seq. ${ }^{4}$ & 7547 & 31.6 & KT223779 & Jelocnik et al., 2015a \\
\hline Marsbar* & Koala, Australia & Culture/UGT & Cystitis & WGS $^{3}$ & 7547 & 31.5 & KT223775 & Bachmann, et al., 2014 \\
\hline IPTaLE* & Koala, Australia & Culture/Ocular & Conjunctivitis & $\mathrm{WGS}^{3}$ & 7547 & 31.5 & KT223772 & Bachmann, et al., 2014 \\
\hline DBDeUG* & Koala, Australia & Culture/UGT & UGT infection & WGS $^{3}$ & 7547 & 31.5 & KT223770 & Bachmann, et al., 2014 \\
\hline HazBoEye & Koala, Australia & Culture/Ocular & Conjunctivitis & WGS $^{3}$ & 7547 & 31.8 & KT352920 & Jelocnik et al., 2015a \\
\hline HazBoUGT & Koala, Australia & Culture/UGT & Conjunctivitis & WGS $^{3}$ & 7547 & 31.8 & KT352921 & Jelocnik et al., 2015a \\
\hline NoHerEye & Koala, Australia & Culture/Ocular & Conjunctivitis & $\mathrm{WGS}^{3}$ & 7547 & 31.5 & KT352922 & Jelocnik et al., 2015a \\
\hline TedHUre & Koala, Australia & Culture/Urethra & Cystitis & WGS $^{3}$ & 7547 & 31.5 & KT352923 & Jelocnik et al., 2015a \\
\hline PMHaUre & Koala, Australia & Culture/Urethra & Cystitis & WGS $^{3}$ & 7547 & 31.5 & KT352924 & Jelocnik et al., 2015a \\
\hline
\end{tabular}


479 *: previously characterised plasmid; ${ }^{1}$ : No clinical disease; ${ }^{2}$ : Sporadic bovine encephalomyelitis. ${ }^{3}:$ Contig from whole genome sequencing; ${ }^{4}:$ Conventional PCR overlapping fragments, dideoxy 480 sequenced. 
481 Table 2. Characteristics of plasmid CDSs from 21 characterised pCpec from C. pecorum pig, cattle, sheep, and koala strains.

\begin{tabular}{|c|c|c|c|c|c|c|c|c|c|}
\hline $\begin{array}{c}\text { Plasmid } \\
\text { CDSs/ } \\
\text { annotation }\end{array}$ & $\begin{array}{l}\text { Predicted } \\
\text { function }\end{array}$ & $\begin{array}{c}\text { Length } \\
\text { (bp)/ } \\
\text { predicted } \\
\text { a.a } \\
\end{array}$ & $\begin{array}{l}\text { No. of non- } \\
\text { synonymous } \\
\text { substitutions }\end{array}$ & $\mathbf{d}_{\mathbf{n}}^{*}$ & $\begin{array}{c}\text { No. of } \\
\text { synonymous } \\
\text { substitutions }\end{array}$ & $d_{s}^{*}$ & $d_{n} / d_{s}$ & $\Delta \mathrm{nt}$ & $\begin{array}{l}\text { No. of } \\
\text { alleles }\end{array}$ \\
\hline $\begin{array}{c}\text { CDS 1/ } \\
p \boldsymbol{G P 8}\end{array}$ & Integrase & $936 / 312$ & 2 & 0.00113 & 5 & 0.00995 & 0.113 & 7 & 4 \\
\hline $\begin{array}{c}\text { CDS 2/ } \\
p G P 8 \\
\end{array}$ & Integrase & $1026 / 342$ & 6 & 0.00278 & 7 & 0.01280 & 0.217 & 13 & 8 \\
\hline $\begin{array}{c}\mathrm{CDS} 3 / \\
\boldsymbol{p} \boldsymbol{G P 1}\end{array}$ & $\begin{array}{c}\text { Replicative } \\
\text { DNA } \\
\text { helicase } \\
\end{array}$ & $1374 / 458$ & 4 & 0.00148 & 7 & 0.01130 & 0.131 & 11 & 6 \\
\hline $\begin{array}{c}\text { CDS } 4 / \\
\boldsymbol{p} \boldsymbol{G P 2}\end{array}$ & $\begin{array}{l}\text { Virulence } \\
\text { plasmid } \\
\text { protein }^{\mathrm{a}}\end{array}$ & $1026 / 342$ & 2 & 0.00074 & 10 & 0.01881 & 0.039 & 12 & 6 \\
\hline $\begin{array}{c}\text { CDS } 5 \text { / } \\
\boldsymbol{p} \boldsymbol{G P 3}\end{array}$ & $\begin{array}{l}\text { Virulence } \\
\text { plasmid } \\
\text { protein }^{\mathrm{a}}\end{array}$ & $795 / 265$ & 4 & 0.00290 & 6 & 0.01219 & 0.131 & 10 & 6 \\
\hline $\begin{array}{c}\mathrm{CDS} 6 / \\
\boldsymbol{p} \boldsymbol{G P 4}\end{array}$ & $\begin{array}{l}\text { Virulence } \\
\text { plasmid } \\
\text { protein }^{\mathrm{a}} \\
\end{array}$ & $309 / 103$ & 0 & 0 & 3 & 0.01654 & 0 & 3 & 4 \\
\hline $\begin{array}{c}\text { CDS } 7 / \\
\boldsymbol{P a r} \boldsymbol{A}\end{array}$ & $\begin{array}{c}\text { Plasmid } \\
\text { partitioning } \\
\text { protein }\end{array}$ & $783 / 261$ & 2 & 0.00097 & 8 & 0.01838 & 0.053 & 10 & 4 \\
\hline $\begin{array}{c}\text { CDS 8 / } \\
\boldsymbol{p} \boldsymbol{G P 6}\end{array}$ & $\begin{array}{l}\text { Plasmid } \\
\text { replication } \\
\text { protein }\end{array}$ & $744 / 248$ & 2 & 0.01839 & 7 & 0.00141 & 0.077 & 9 & 6 \\
\hline $\begin{array}{c}\text { Intergenic } \\
\text { region } \\
\text { (between } \\
\text { CDSs } 8 \text { and } 1 \text { ) } \\
\end{array}$ & $\begin{array}{l}\text { Origin of } \\
\text { replication }\end{array}$ & $\begin{array}{c}207 / \\
4 \times 22 \mathrm{bp} \\
\text { tandem } \\
\text { repeats }\end{array}$ & n.a & n.a & n.a & n.a & n.a & 2 & 3 \\
\hline
\end{tabular}


484 Figure 1. Graphical representation of the $\mathrm{pCpecL1}$ and annotated CDSs, including primer locations. Putative ori at the top. The 22bp 485 tandem repeat units are indicated by blue arrows.

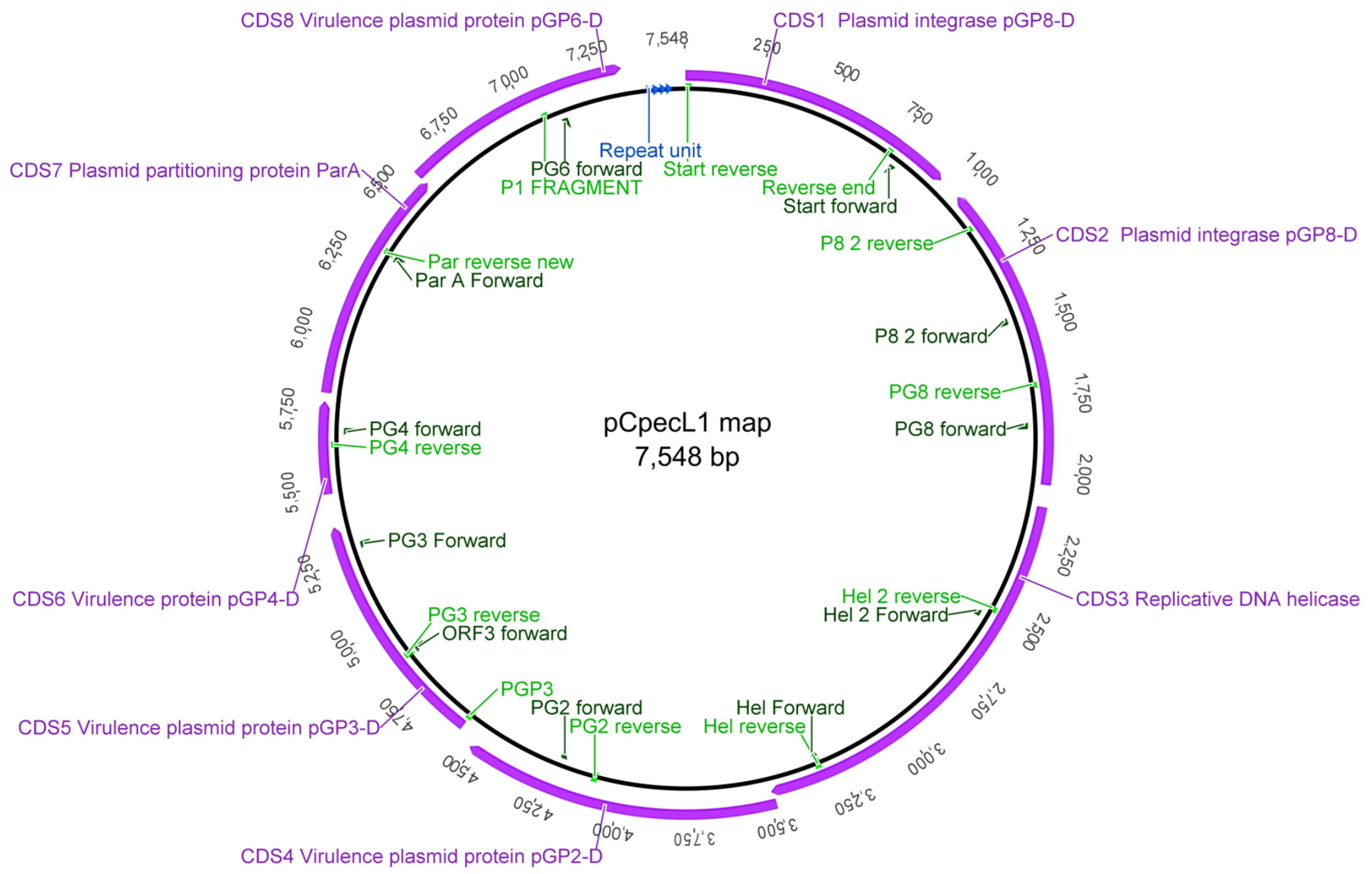


Figure 2. Bayesian phylogenetic analyses of A) 7.5kbp $21 \mathrm{pCpec}$ sequences from C. pecorum strains from porcine, ovine, bovine, and koala hosts; and B) concatenated sequences of the seven MLST C. pecorum genes from 18 corresponding strains harbouring plasmids. Posterior probabilities $>0.75$ are displayed on the tree nodes, while the hosts are indicated by the colouring on the legend.

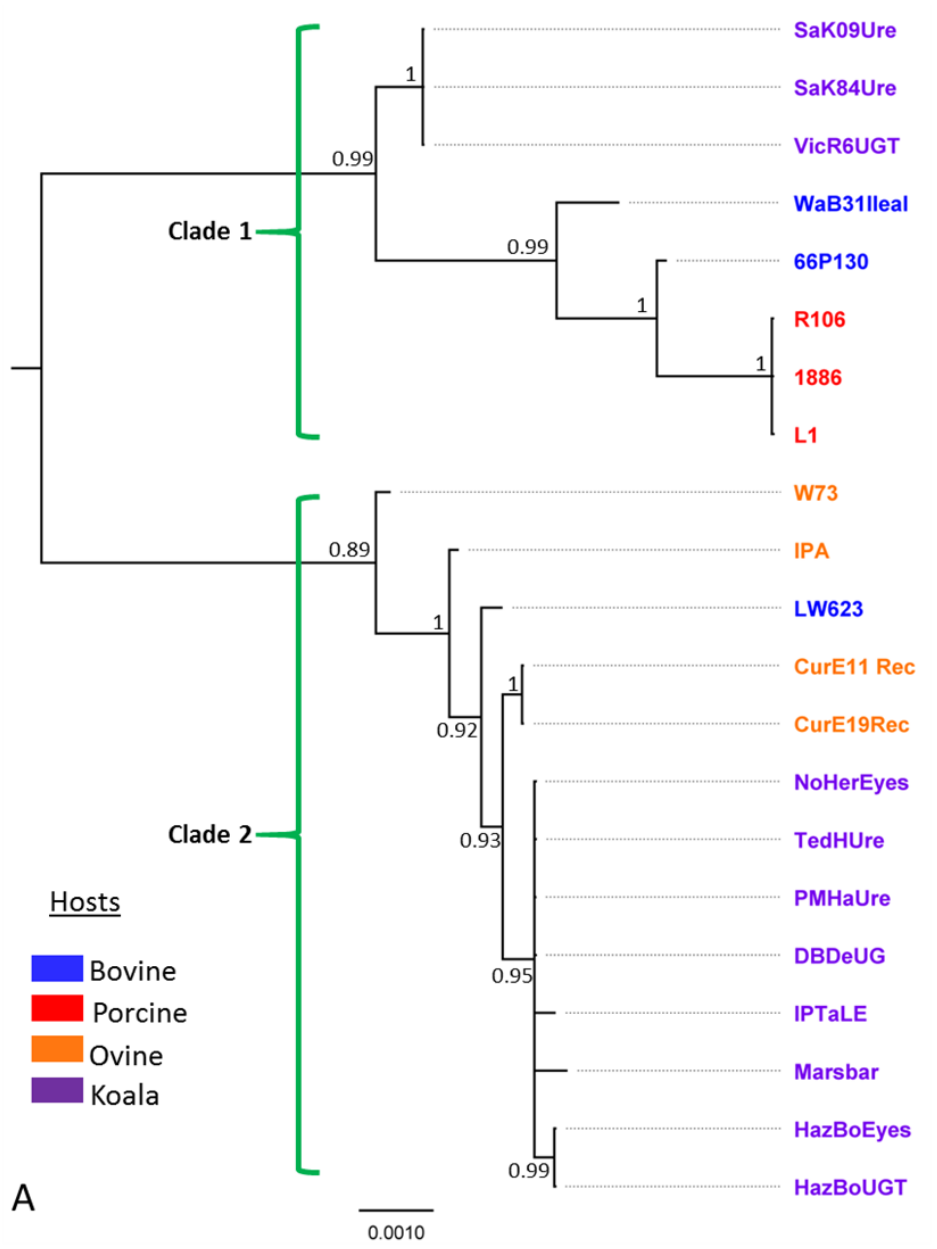

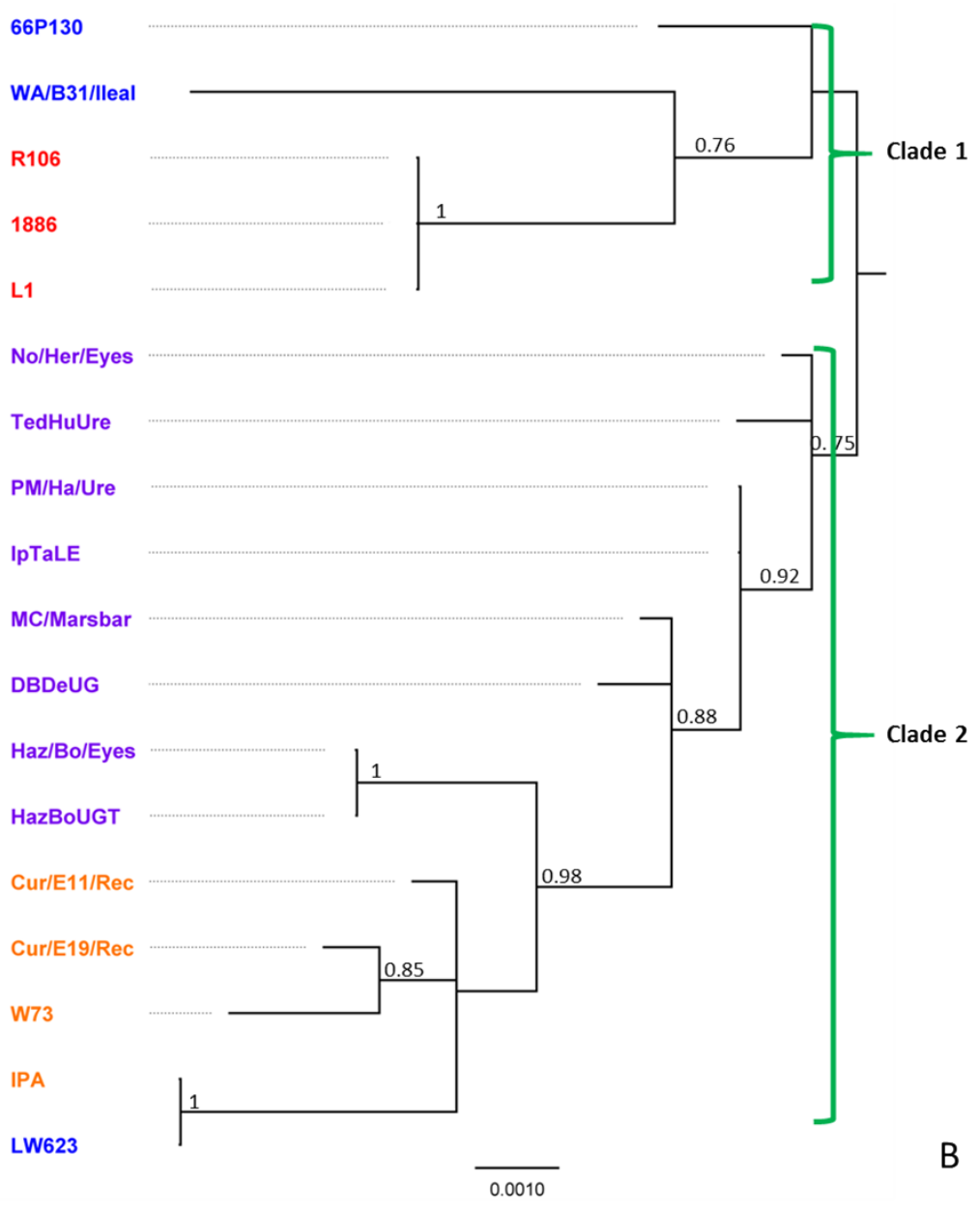

\section{Supplemental data}


Table S1. Primers used for generating plasmid $(\mathrm{p} C p e c)$ fragments and their characteristics.

Figure S1. Bayesian phylogenetic analyses of A) plasmid sequences from eight related chlamydial species, compared to the B) $16 \mathrm{~S}$ rRNA gene sequences from corresponding chlamydial strains harbouring these plasmids. Posterior probabilities $>0.75$ are displayed on the tree nodes. C. muridarum sequences were used as an out-group. Associated plasmid and 16S rRNA gene sequence from the same chlamydial strain are denoted by coloured arrows.

Figure S2. A) SNP distribution in the pCpec genotypes, using Genotype A as a reference. SNP positions are highlighted in black, while the type of variants are highlighted in purple for A, pink for G, green for C, light blue for T. SNPs resulting in non-synonymous changes are indicated with red boxes. A single bp insertion in the $\mathrm{pCpec}$ genotypes I, J and L are indicated with a green box. The $22 \mathrm{bp}$ tandem repeat units are indicated by blue arrows. B) The pCpec phylogeny aligned to the tracks of pCpec SNP only alignment, using reconstructed plasmid sequence $\mathrm{N} 1$ as a reference. Above the alignment is the graphical representation of $\mathrm{p} C p e c \mathrm{CDSs}$ position in reference to the SNPs alignment, while the top line is numbering the successive SNPs as detected in the pCpec sequences. SNPs are highlighted as disagreements to the reference sequence. Homoplasic SNPs are denoted with star symbols. Ones resulting in a nonsynonymous change are denoted with red stars, while the ones resulting in a synonymous change are denoted with blue stars.

Figure S3. Neighbor-Joining phylogenetic analyses of the $21 \mathrm{pCpec}$ sequences from C. pecorum strains from porcine, ovine, bovine, and koala hosts using C. pneumoniae pLPCoLN as an out-group. Bootstrap values (1000 times repetitions) are displayed on the tree nodes. 